\title{
APPLICATION OF FUZZY LOGIC IN TRANSPORT PlanNING
}

\author{
Amrita Sarkar ${ }^{1}$ G.Sahoo ${ }^{2}$ and U.C.Sahoo ${ }^{3}$ \\ ${ }^{1}$ Research Scholar, Department of Information Technology, B.I.T Mesra, Ranchi \\ amrita.mannaegmail.com \\ ${ }^{2}$ Professor and Head,Department of Information Technology, B.I.T, Mesra, Ranchi \\ gsahoo@bitmesra.ac.in \\ ${ }^{3}$ Assistant Professor, Department of Civil Engineerng, I.I.T, Bhabaneswar \\ ucsahooditbbs.ac.in
}

\begin{abstract}
Fuzzy logic is shown to be a very promising mathematical approach for modelling traffic and transportation processes characterized by subjectivity, ambiguity, uncertainty and imprecision. The basic premises of fuzzy logic systems are presented as well as a detailed analysis of fuzzy logic systems developed to solve various traffic and transportation planning problems. Emphasis is put on the importance of fuzzy logic systems as universal approximators in solving traffic and transportation problems. This paper presents an analysis of the results achieved using fuzzy logic to model complex traffic and transportation processes.
\end{abstract}

\section{KEYWORDS}

Fuzzy Logic, Transportation Planning, Mathematical modelling

\section{INTRODUCTION}

Transportation planning is a wide human-oriented field with diverse and challenging problems waiting to be solved. Characteristics and performances of transport systems - services, costs, infrastructures, vehicles and control systems are usually defined on the basis of quantitative evaluation of their main effects. Most of the transport decisions take place under imprecision, uncertainty and partial truth. Some objectives and constraints are often difficult to be measured by crisp values. Traditional analytical techniques were found to be non-effective when dealing with problems in which the dependencies between variables are too complex or ill-defined. Moreover, hard computing models can not deal effectively with the transport decision-makers' ambiguities and uncertainties.

In the past several decades a variety of deterministic and stochastic models have been developed to solve complex traffic and transportation engineering problems. These mathematical models use different formulae and equations to solve such problems. During their education and training, engineers are most often directed to the use of exclusively objective knowledge (formulae and equations). However, when solving real-life engineering problems, linguistic information is often encountered that is frequently hard to quantify using 'classical' mathematical techniques. This linguistic information represents subjective knowledge (linguistic information). 
The primary goal of this paper is the applications of fuzzy logic in traffic and transportation engineering, and to indicate the directions for future research in this area.

\section{FUZZY LOGIC SYSTEMS FOR TRANSPORTATION}

Basic results linked to the development of fuzzy logic date back to Zadeh (1973) [51] and Mamdani and Assilian (1975) [24]. Introducing a concept he called 'Approximate Reasoning', Zadeh successfully showed that vague logical statements enable the formation of algorithms that can use vague data to derive vague inferences. Zadeh assumed his approach would be beneficial above all in the study of complex humanistic systems.

What is a fuzzy logic system? Mendel (1995) [25] explains the concept of a fuzzy logic system-a (FLS) as follows: 'In general a FLS is a nonlinear mapping of an input data (feature) vector into a scalar output (the vector output case decomposes into a collection of independent multi-input/ single-output systems)'.

Pappis and Mamdani (1977) [28] published the first paper in which a practical traffic and transportation problem was solved using fuzzy logic. In the mid- and late-1980s, a group of Japanese authors made a significant contribution to fuzzy set theory applications in traffic and transportation. Nakatsuyama et al. (1983) [27], Sugeno and Nishida (1985) [36] and particularly Sasaki and Akiyama, (1986, 1987, 1988) [33, 34, 35] solved complex traffic and transportation problems indicating the great potential of using fuzzy set theory techniques. At the end of the 1980s and beginning of the 1990s, the fuzzy set theory in traffic and transportation became extensively used at American universities. The pioneering work of the research team of the University of Delaware deserves special attention, headed by Professor Shinya Kikuchi (Chakroborthy, 1990 [5]; Chakroborthy and Kikuchi, 1990 [6]; Perincherry, 1990 [29]; Perincherry and Kikuchi, 1990 [30]; TeodorovicÂ and Kikuchi, (1990, 1991) [41, 42]; Kikuchi et al.,(1991, 1993) [19, 20]; Kikuchi, 1992 [18]). In the early and mid-1990s, interest in fuzzy logic applications in traffic and transportation increased in other world universities. Different traffic and transportation problems successfully solved using fuzzy set theory techniques were presented in the works of Chen et al. (1990) [8], Tzeng et al. (1996) [44], Lotan and Koutsopoulos

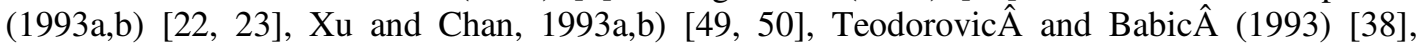
Akiyama and Shao (1993) [1], Chang and Shyu (1993) [9], Chanas et al. (1993) [7], Akiyama amd Yamanishi (1993) [2], Deb (1993) [10], Perkinson (1994) [31], Hsiao et al. (1994) [12], Vukadinovic $\hat{A}$ and TeodorovicÂ (1994) [47], Teodorovic $\hat{A}$ et al. (1994) [39], TeodorovicÂ

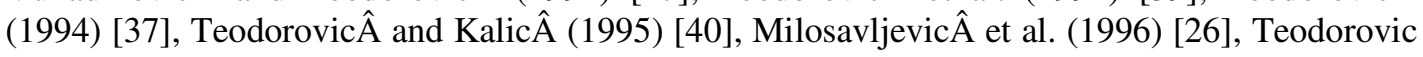
and Pavkovic (1996) [43] and Tzeng et al. (1996) [44].

Let us try to explain why fuzzy logic systems were developed for transportation engineering. Great many of the problems in the field of transportation planning and traffic control are often ill defined, ambiguous and vague. As already mentioned, many traffic and transportation problems, phenomena and parameters are characterized by subjectivity. It is hard to disregard the fact that subjective judgment is present in problems dealing with the choice of route, mode of transportation and carrier, a driver's perceptions and reactions, an established level of service, defining safety standards, defining criteria to rank alternative transportation plans and projects, etc. It should also be emphasized that both deterministic and stochastic models that have been developed to solve a variety of complex traffic and transportation problems are characterized by mathematics based on binary logic. Without denying the importance of binary logic as the basis for the development of many scientific disciplines and technology leading to the prosperity of man's society, we must note that it cannot deal effectively with passengers', dispatchers' or drivers' feelings of uncertainty, vagueness and ambiguity. Since the fuzzy set theory recognizes the vague boundary that exists in some sets, different fuzzy set theory techniques need to be used 
in order to properly model traffic and transportation problems characterized by ambiguity, subjectivity and uncertainty.Some applications of Fuzzy Logic in transport planning are discussed below.

\subsection{Trip generation}

Trip generation constitutes the first stage in the traditional transportation planning process. Trip generation defines that how many people want to go out of their homes and what their purposes are. Researchers emphasized that more non-congestion trip generation models have better prediction and adaptation ability. So in many years to validate different ideas, experts have invented variety of models and they always try to estimate the number of generated trips in a better method helping newer model presentation. Also they try to decrease the previous models problems and defects as much as possible.

Trip generation problem was solved using fuzzy logic by Kalic and Teodorovic (1997a, b) [16, 17]. Fuzzy rule base was generated by learning from numerical examples. For this purpose, the procedure proposed by Wang and Mendel (1992a, b, c) [46, 47, 48] was used. Firstly, the available set of data was divided into two subsets: the first was used for generation of the fuzzy rule base, and the other was intended to be a control data subset. After the fuzzy rule base was created, the obtained fuzzy system was tested on both subsets of data. The estimate of the number of trips for the subsets was also determined using artificial neural networks, as well as by multiple linear regressions. The training of the neural network was done using simulated annealing technique. After the testing, the fuzzy logic approach proved to give the closest estimate of the actual number of trips generated in a given area.

One of the most important problems in the field of transportation planning is the problem of Origin-destination estimation from link counts. In order to decrease the cost of passenger surveys, traffic count are undertaken on certain links of the transportation network. In the next phase an origin-destination matrix is estimated from the link counts. In the last two decades a greater number of models have been developed for origin-destination estimation from link counts. Xu and Chan (1993a, b) $[49,50]$ were the first to use the fuzzy set theory techniques when analyzing the problems arising from the poor quality of link count data. As known, traffic counts are often subject to errors. Xu and Chan $(1993 \mathrm{a}, \mathrm{b})[49,50]$ particularly pointed out the problem of traffic counts in countries such as China in which mixed urban traffic flow with heavy bicycle volume is common. In such situations the heavy bicycle volume also makes precise vehicular counts very difficult. Xu and Chan (1993a, b) [49, 50] estimated an origin-destination matrix with fuzzy weights.

\subsection{Trip distribution}

Trip distribution constitutes the second stage in the traditional transportation planning process. Trip distribution models are used to determine the number of trips between pairs of zones when the number of trips generated attracted by particular zones is known. Thus, the prediction of trip distribution involves the prediction of flows in a network regardless of a possible transportation mode or travel route.

Traffic flows and trip distribution resulted from human choices that are affected by social and individual variables of the commuters. Due to this fact that human decision making are more consistent with fuzzy logic in comparison with crisp mathematics, it seems that fuzzy logic could be a logical tool to map such areas. Modelling a trip distribution system with fuzzy inference systems would enjoy the exploration of subjective pattern of decision makers. Meanwhile, one of the most important parts of forecasting procedure is to predict the trips flow between a given 
origin-destination pair. Different models have been developed to forecast trip flows in recent years. The key to a successful forecasting is to recognize the existing patterns correctly. Trip distribution problem was also solved using fuzzy logic by Kalic and Teodorovic (1996, 1997a, b $[15,16,17]$ Like in the case of trip generation problem, Kalic and Teodorovic (1996) [15] generated fuzzy rule base by learning from numerical examples.

A three phases fuzzy inference system (FIS) was proposed by Jassbi et al.(2011) [14] to map social and demographic variables to total number of trips between origin-destination (OD) pairs. Fuzzy rule bases in the model are in fact the exploration of transportation experts' subjective patterns.

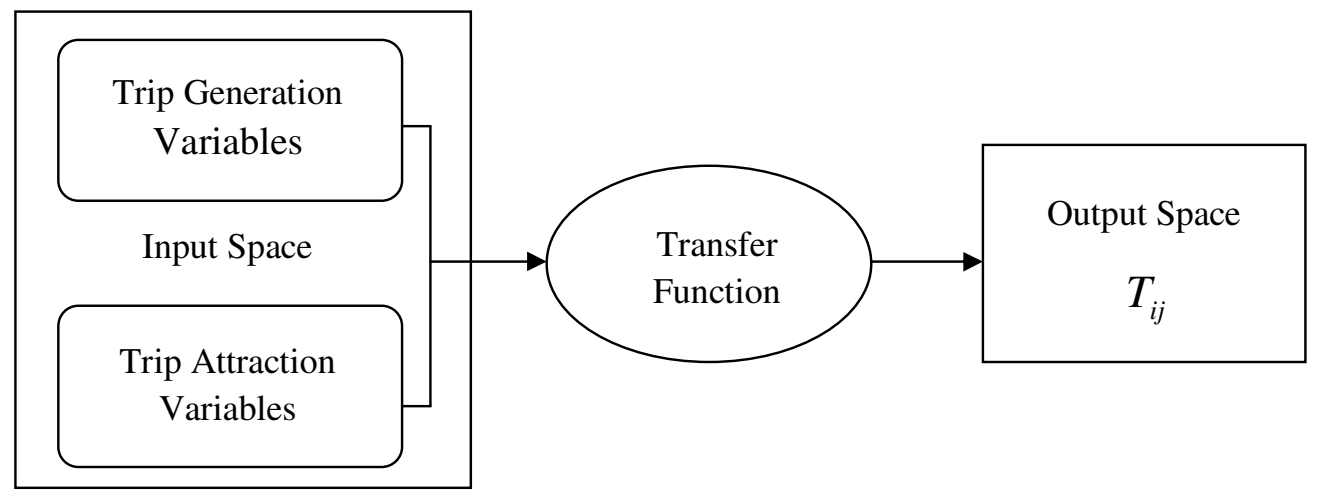

Figure 1. Conceptual model of trip forecasting input-output space mapping

Figure 1 illustrates the conceptual framework of the trip forecasting problem. The main concern of the problem was to approximate the transfer function that is capable of mapping trip attraction and generation variables to number of trips between two given regions. As it addressed in introduction section, Different kinds of transfer functions had been used in this problem. Here, a fuzzy inference system is used to map input space to output space.

The process of fuzzy inference involves:

i. Defining If-Then rules

ii. Defining membership functions

iii. Appling logical operations

There are two types of fuzzy inference systems that usually can be implemented: Mamdani-type and Sugenotype. These two types of inference systems vary somewhat in the way outputs are determined.

Input space is 'Trip Generation variables and Trip attraction variables'. These are the variables that have direct impacts on the generation and attraction of trips of any given region. Output space is 'the number of trips between two given regions'. The number of trips would be approximated by a transfer function that is formed based on the relations of trip generation capabilities of origin and trip attraction capacities of destination. 
International Journal on Soft Computing (IJSC) Vol.3, No.2, May 2012

Table 1. Trip generation variables (input space variables)

\begin{tabular}{|c|l|}
\hline Input space variables & \multicolumn{1}{|c|}{ Generation variables } \\
\hline$I_{1}$ & Occupation in the residence area \\
\hline$I_{2}$ & Population \\
\hline$I_{3}$ & Residential space \\
\hline$I_{4}$ & Population density \\
\hline$I_{5}$ & Number of households \\
\hline$I_{6}$ & Car ownership rate \\
\hline$I_{7}$ & Average price of one square meter of land \\
\hline$I_{8}$ & Students population \\
\hline$I_{9}$ & Traffic zone space \\
\hline$I_{10}$ & Number of residential buildings \\
\hline$I_{11}$ & Distance to entertainment complexes \\
\hline & \\
\hline & \\
\hline & \\
\hline & \\
\hline
\end{tabular}

Table 2. Trip attraction variables (input space variables)

\begin{tabular}{|c|l|}
\hline Input space variables & \multicolumn{1}{|c|}{ Attraction variables } \\
\hline$I_{1}$ & Occupation in the working area \\
\hline$I_{2}$ & $\begin{array}{l}\text { Business/ Administrative/Agricultural/Industrial } \\
\text { Land Space }\end{array}$ \\
\hline$I_{3}$ & Administrative building space \\
\hline$I_{4}$ & $\begin{array}{l}\text { Number of Administrative/Business/Industrial } \\
\text { Buildings }\end{array}$ \\
\hline$I_{5}$ & Schools' space \\
\hline$I_{6}$ & Number of Students/Schools/Classes \\
\hline & \\
\hline
\end{tabular}


International Journal on Soft Computing (IJSC) Vol.3, No.2, May 2012

\begin{tabular}{|c|l|}
\hline$I_{7}$ & Number of Universities/Students \\
\hline$I_{8}$ & Number of Retailers \\
\hline$I_{9}$ & $\begin{array}{l}\text { Number and Capacity of Cinemas/Mosques/ } \\
\text { Exhibitions/ }\end{array}$ \\
\hline$I_{10}$ & Parks/Hospitals \\
\hline
\end{tabular}

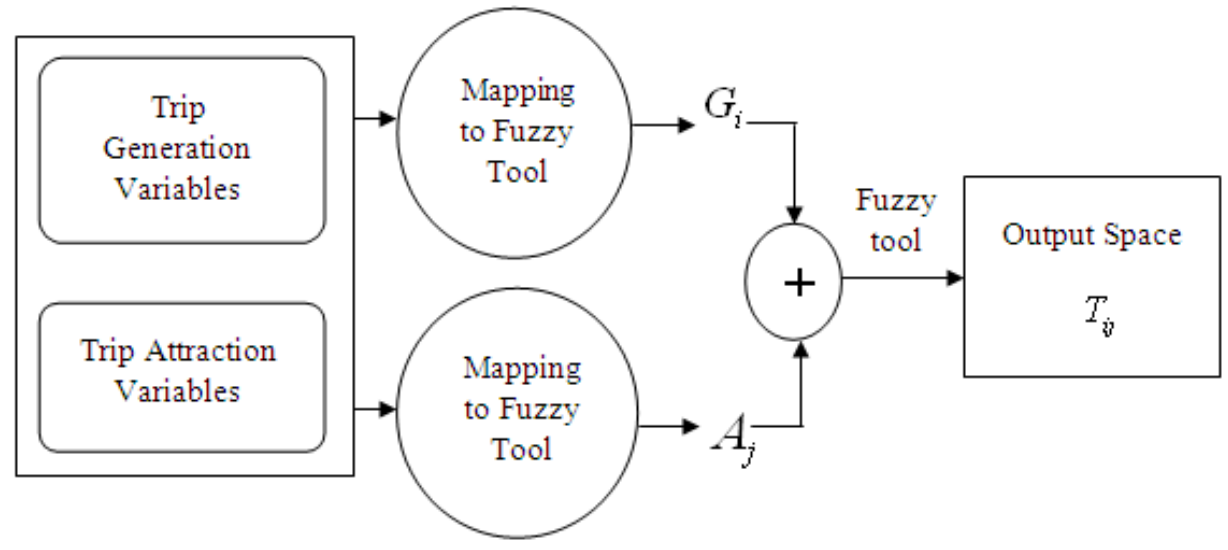

Figure 2. Conceptual fuzzy model: Mapping trip generation and attraction variables to total number of trips between two given regions

To map input space to output space, different fuzzy scenarios could be considered. Figure 2 above illustrates the framework of conceptual fuzzy model that is used to forecast the number of trips in this work. As it illustrated in above, three independent fuzzy inference systems are used. The first FIS is aimed to map generation variables to number of generated trips by the given region $\left(G_{i}\right)$.The second FIS is designed to map attraction variables to number of attracted trips by the given region $\left(A_{j}\right)$. At the end, the third FIS is designed to combine the output of the first and second FIS for aggregation of two given regions $G_{i}$ and $A_{j}$ and calculating the final total number of trips between two regions $\left(T_{i j}\right)$.

\subsection{Modal split}

The third phase of transport planning named as modal split is intended to know which modes of transportation (bus, train etc.) are used by how much fraction of people. Teodorovic and Kalic (1996) used fuzzy logic to solve the mode choice problem. The authors illustrated the possibilities of fuzzy logic in solving the mode choice problem using a hypothetical numerical example. The fuzzy rule base was generated using available numerical data on the differences between the travel times and travel costs of competitive modes. In other words, learning from examples generated the fuzzy rule base. The technique proposed by Wang and Mendel (1992a) was used to generate the fuzzy rule base by learning from examples. There was extremely good agreement between the results obtained using fuzzy logic and real results. 
Quadrado and Quadrado (1996) [32] used fuzzy logic to determine the accessibility of different transportation modes in the Lisbon Metropolitan Area. The authors first pointed out that all variables used in the 'classical' way of calculating accessibility are characterized by fuzziness. A fuzzy rule base was developed for each mode of transportation. Accessibility was shown as a percentage between 0 and $100 \%$. One of the typical rules to calculate accessibility reads:

If the number of people involved in movements is VERY BIG and the time used in those movements is VERY SMALL

Then the accessibility is HYPER BIG

The authors thus put an end to the tradition of treating accessibility as a deterministic concept and offered the possibility of a better representation of accessibility.

The purpose of modal split step is to determine the share of traffic of vehicle and Public transportation means in urban area. One of the most well-known model that called "Logit model" is often used.

$$
q=\bar{q}_{r s} \frac{1}{1+\exp \left(-\theta\left(C_{r s}^{\text {tran }}-C_{r s}\right)\right)}
$$

This is a sort of nonlinear function model that based on random utility theory. In this formulation, the share of mass transit between the OD pair (r-s) is determined by the general cost of mass transit $C_{r s}^{\text {tran }}$ and general cost of $\operatorname{car} C_{r s}$.

The outline of the triangular fuzzy number for each link travel time is illustrated in Figure 8 below. Figure 3 presents an example of a fuzzy number with a triangular member-ship function corresponding to the label "travel time difference is medium".

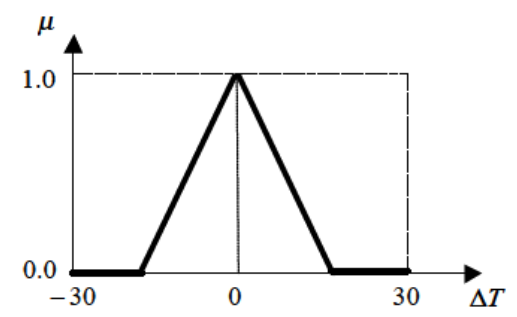

Figure 3. Description of fuzzy travel time difference $\Delta T=C_{r s}^{\text {Transit }}-C_{r s}$

where $\Delta T$ is the time difference on each OD pair, $C_{r s}^{\text {Transit }}$ is mass transit travel time, $C_{r s}$ is car travel time are denoted.

As mentioned earlier, when attempting to represent a mode choice model that considers about human behavior, the fuzzy reasoning is applied. Here are three simple rules that used in this model:

Rule 1: If $\Delta T$ is big, then Transit $P_{\text {Transit }}$ is big.

Rule 2: If $\Delta T$ is medium, then Transit $P_{\text {Transit }}$ is medium. 
Rule 3: If $\Delta T$ is small, then Transit $P_{\text {Transit }}$ is small.

where $P_{\text {Transit }}$ is the rate of transit.

Here, in each OD pair, the value of the linguistic variable "big", "medium" and "small" will be defined. Each membership function of linguistic variable is summarized in Figure 4.

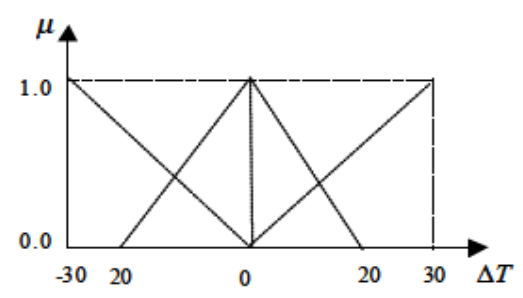

Figure 4. Membership function

Each OD pair has a range of travel time difference, the ranges of some representative OD pairs is listed in table 3 . Figure 9 shows the membership function of an OD pair and $(-30,30)$ is the range of travel time difference for the objective OD pair. In this description, the travel time difference is a crisp value. This is also the medium rule in fuzzy rule sets that supposed in this model.

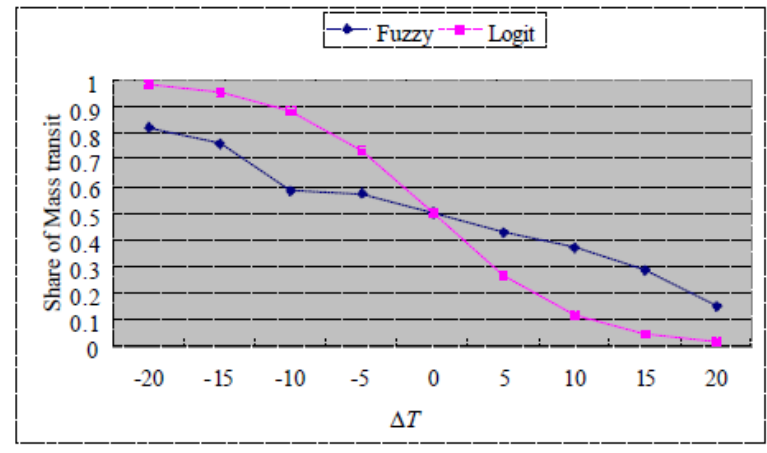

Figure 5.The share of mass transit

Figure 5 presents the change of mass transit's share and the time difference in one OD pair, assume that the range of travel time difference is $(-20,20)$. The results obtained from both models, logit mode choice model and Fuzzy mode choice model are shown in the figure, the share of mass transit share computed by logit mode choice model changes rapidly when the absolute value of travel time difference is small, in contrast to that computed by Fuzzy mode choice model.

For each OD pair, the time different $(\Delta T)$ between mass transit travel time and car travel time of iteration number $\mathrm{n}$ will be calculated by the combine modal in loop number $\mathrm{n}-1$, until the condition is met. For each OD pair, the range is defined before hand, and they are listed in table 3. Because it becomes too long to list the ranges of all the OD pairs in the network, in the table, only some representative OD pairs generated from origin zone 1, 11 are listed. 


\begin{tabular}{|c|c|c|}
\hline $\begin{array}{c}\text { Origin } \\
\text { Zone }\end{array}$ & $\begin{array}{c}\text { Destination } \\
\text { Zone }\end{array}$ & Range \\
\hline 1 & 2 & 20 \\
\hline 1 & 3 & 20 \\
\hline 1 & 4 & 40 \\
\hline 1 & 5 & 40 \\
\hline$\vdots$ & $\vdots$ & $\vdots$ \\
\hline 11 & 7 & 60 \\
\hline 11 & 8 & 60 \\
\hline 11 & 9 & 30 \\
\hline 11 & 10 & 20 \\
\hline
\end{tabular}

Table 3. Zone pairs and ranges

As mentioned above, shares of Car and mass transit will be estimated by modal split with fuzzy reasoning, in this section, the "Min - Max - Gravity "method is applied. The method is simply represented as figure 6. For example, in this figure, the range of one OD pair is defined as 20 minutes, the time different is 6 minutes, the share of Car is $63 \%$ and share of mass transit is $27 \%$.

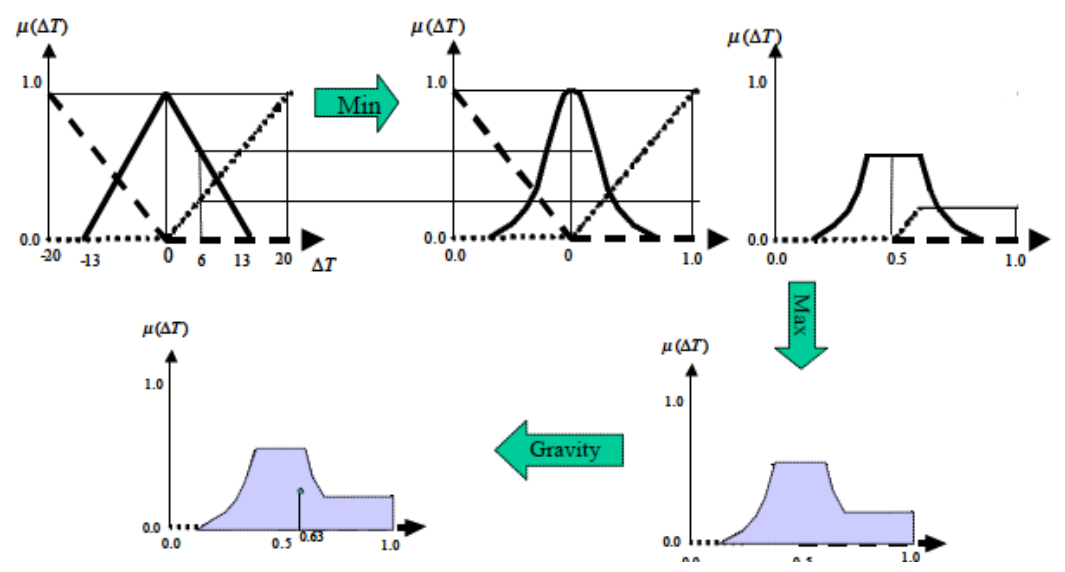

Figure 6. The "Min - Max- Gravity" method

\subsection{Route choice}

In the past four decades, the route choice problem, along with the traffic assignment problem, has been considered by a large number of authors worldwide. Teodorovic and Kikuchi (1990) [41] were first to model the complex route choice problem using fuzzy logic. They used fuzzy inference techniques to study the binary route choice problem. Akiyama et al. (1993) [2] also developed a model for route choice behavior based on the fuzzy reasoning approach. Lotan and Koutsopoulos (1993a, b) [22, 23] developed models for route choice behavior in the presence of information based on concepts from approximate reasoning and fuzzy control. The research of Lotan and Koutsopoulos (1993a, b) [22, 23] is particularly important within the context of ongoing research in Intelligent Vehicle Highway Systems (IVHS). Teodorovic and Kalic (1995) [40] developed an approximate reasoning algorithm to solve the route choice problem in air transportation. Akiyama and Tsuboi (1996) [4] studied route choice behavior by multi-stage fuzzy reasoning. 
Let us consider the simplest case when the user is to choose one of two possible paths between the origin and destination of movement. Perceived travel time is very often 'fuzzy' and can be treated as a fuzzy set. In other words, when subjectively estimating travel time between two points, expressions are used such as 'it takes about 20 minutes from point $\mathrm{C}$ to point $\mathrm{D}$ ', 'you'll get there in about half an hour', etc. it is assumed that users choose their paths based on a comparison of the characteristics of alternative paths. Despite the fact that travel time is a measurable parameter, a driver's notion of travel time when he/she makes the route choice is often fuzzy. Thus, driver's preference for a route is based on a comparison of two fuzzy numbers. As a result, his/her certainty of choosing a route is also not crisp. The route choice model can represent the decision process under two layers of uncertainty: driver's judgment of time and driver's preference (Teodorovic and Kikuchi, 1990) [41].

Let us denote by TA and TB, respectively, fuzzy sets representing estimated travel time (by one user) along path $\mathrm{A}$ and path $\mathrm{B}$. When comparing travel times along paths $\mathrm{A}$ and $\mathrm{B}$, the user might estimate, for example, that travel time along path A, TA is 'much shorter than TB', 'shorter than TB', 'equal to TB', 'longer than TB', and 'much longer than TB'. These fuzzy sets are denoted in the following manner: MLTB-'much less than TB', LTB-'less than TB', GTB-'greater than TB', MGTB-'much greater than TB'. Let us assume that a user has a specific preference regarding the choice of each of the possible paths through the network. This preference can be 'stronger' or 'weaker'. Let us introduce into the discussion a preference index that can take values on the interval from 0 to 1 . When the user has an absolute preference for a specific path we consider the preference index to be equal to 1 .

This preference index decreases along with a decrease in the strength of the preference. Fuzzy sets such as 'very strong', 'strong', 'medium' can express the strength of the user's preference, 'weak' and 'very weak'. The following rules of approximate reasoning are proposed to establish the transport network user's preference strength:

$\begin{array}{llll}\text { If } & \text { TA=MLTB } & \text { then } & \text { PA=VERY STRONG } \\ \text { If } & \text { TA=LTB } & \text { then } & \text { PA=STRONG } \\ \text { If } & \text { TA=TB } & \text { then } & \text { PA=MEDIUM } \\ \text { If } & \text { TA=GTB } & \text { then } & \text { PA=WEAK } \\ \text { If } & \text { TA=MGTB } & \text { then } & \text { PA=VERY WEAK }\end{array}$

where PA is the preference index associated with path $\mathrm{A}$.

An approximate reasoning algorithm is used to establish the preference strength for every network user. Once the preference index has been determined for each network user, an algorithm must be developed that will determine the number of users along individual links. Such algorithms are known in the literature as network 'loading' algorithms developed a network 'loading' algorithm is developed in which the basic input data are the preference indexes of individual network users. It is assumed that the perceived travel times are distributed around a previously measured most likely travel time. The 'loading' algorithm is based on the concept of hybrid numbers (Teodorovic and Kikuchi 1990) [41].

Multi-stage fuzzy reasoning is used to describe the driver decision making process on road networks. Their term paper considers the multi-route choice problem. The first step for multiroute choice is made a survey and collected data. Experiment participants were asked about the number of alternative routes they use going from their origin to their destination (the authors asked the respondents to cite at most three alternatives they may use). For each route considered they defined its characterizing factors (travel time, degree of congestion and risk of accidents). All the participants estimated the values of all the factors using their own perception and 
experience. Appropriate fuzzy numbers could then represent these values. The third survey question dealt with the values of the utilities of the nominated routes. The authors assumed the existence of two stages in the driver decision making process. The firrst approximate reasoning algorithm they proposed determined the utilities of the alternative routes (Akiyama and Tsuboi, 1996) [4]. A typical rule in this approximate reasoning algorithm reads:

If $\quad \mathrm{T}$ is large and CR is small and RA is medium

Then $\mathrm{V}$ is small

where:

(a) $\mathrm{T}=$ perceived travel time

(b) $\mathrm{CR}=$ congestion of a route

(c) RA=risk of accident

(d) V=utility for the route.

The first input variable DF in the second approximate reasoning algorithm is the difference between the utilities associated with the shortest path and the second shortest path. The second input variable DS is the difference in the utilities between the second shortest path and the third shortest path. The third input variable $\mathrm{N}$ refers to the number of alternative routes. When $\mathrm{N}=2$, variable DS does not appear in the premises. A typical rule of the second approximate reasoning algorithm reads:

\section{If $\quad \mathrm{N}=3$ and DF is medium and DS is medium}

Then $\mathrm{FR}(1)$ is large

$\mathrm{FR}(2)$ is medium

$\mathrm{FR}(3)$ is medium

where:

(a) $\mathrm{DF}=$ difference of utilities between the shortest path and the second shortest path

(b) DS=difference of utilities between the second shortest path and the third shortest path

(c) FR(i)=degree of frequency for route $\mathrm{i}$

In Fuzzy Logic Model, membership functions of input and output parameters are determined based on survey results of a particular area and by the help of an expert's knowledge. For input parameters (travel time, traffic safety, congestion and environmental effects), triangular and trapezoidal membership functions are used, while for output parameter (route utility), triangular is used (Figure 7). Most convenient membership functions are defined by examination of statistical dispersion of the survey results and after some trials. 

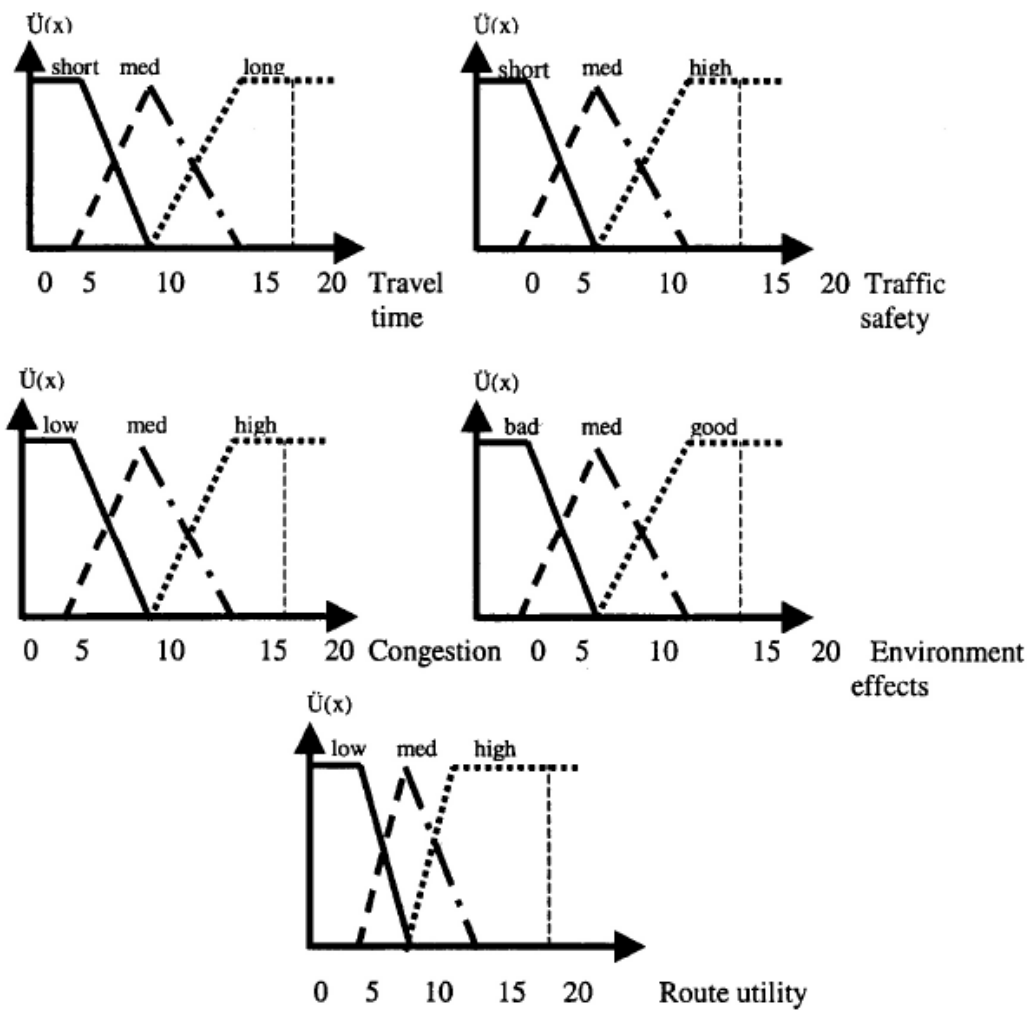

Figure 7. Fuzzy logic route choice model parameters and membership functions

Input parameters that are obtained from survey study were used in the first stage, given values of these parameters are converted to fuzzy using membership functions in the second stage, the rule base was used for inference in the third stage, using rule base a fuzzy output that shows the utility of route was obtained and the fuzzy output was converted to crisp numbers using a deffuzzification method in the fourth stage. At the end, route utility was obtained as a crisp number. After these processes, choice probabilities of routes were determined by multinomial logit formula.

Rule base used in the fuzzy logic model is formed after statistical analysis of the input and output parameters. Rule base consists of $3 * 3 * 3 * 3=81$ rules; 3 for traffic safety; 3 for congestion, 3 for travel time and 3 for environmental effects. These IF-THEN rules are collated with AND operator. Samples of fuzzy logic route choice model rule base are:

IF TT is Short and TS is High and Co is Low and EE is Good THEN RU is High IF TT is Short and TS is Med.and Co is Low and EE is Good THEN RU is High IF TT is Short and TS is Med. and Co is Med and EE is Med THEN RU is Medium IF TT is Long and TS is Med. and Co is Low and EE is Med THEN RU is Medium IF TT is Med. and TS is High and Co is Low and EE is Med.THEN RU is Med. IF TT is Long and TS is Low and Co is High and EE is Med THEN RU is Low

The model output is gained where Mamdani inference mechanism is used. In order to compare fuzzy results of Fuzzy Logic Model with crisp results of mathematical models, fuzzy output is defuzzified using Centroid method as: 


$$
z^{*}=\frac{\int \ddot{u}_{c}(z) \cdot z d z}{\int \ddot{u}_{c}(z) d z}
$$

where, $\mathrm{z}=$ crisp value(value for $\mathrm{x}$ axis), $u_{c}=$ membership value for given crisp value.

\subsection{Traffic assignment}

Many papers devoted to the traffic assignment problem were published in world literature. There are two characteristics common to most of these papers: they are based on Wardrop's principle and they are travel time crisp. Akiyama et al. (1994) observed that 'in real world, however, the driver can only use fuzzy traffic information even if several types of information are available'. These authors succeeded in formulating the fuzzy user equilibrium with fuzzy travel time. They confirmed their assumption about drivers' perception of time as triangular fuzzy numbers by organizing the survey on the network comprised of Hanshin Expressway and urban streets in the Osaka area. In their paper, the authors introduced 'the descriptive method of route choice behavior to design the traffic assignment model'. They showed that 'the state of user equilibrium is also generated even if fuzziness of travel time exists'. Akiyama et al. (1994) [3] developed Fuzzified Frank-Wolfe algorithm to solve the problem considered. This pioneer paper undoubtedly presents a significant study on the relationship between traffic information and drivers' behavior.

\subsubsection{Fuzzy Sets of Perceived Link Travel Time}

The main concern of drivers traversing in the road networks is travel time, travel time of each link is the basic component for assigning traffic. The fuzzy sets of perceived link travel time will be based on deterministic link travel time and actual link travel time

\section{a. Deterministic Link Travel Time}

Deterministic link travel time is determined by deterministic features of the link, like link length, capacity, etc., and also the current deterministic traffic conditions, like traffic flow on this link. The following equation (1) is usually chosen for computing the deterministic link travel time:

$c_{a}(t)=c_{a}\left[u_{a}(t), x_{a}(t)\right]=T_{a, f}\left[1+\beta\left(\frac{u_{a}}{C_{a}}+\frac{x_{a}}{x_{a, \max }}\right)^{\alpha}\right] \forall a$

where $\alpha$ and $\beta$ are coefficients; $T_{a, f}$ is the free flow travel time on link $a ; u_{a}$ is its inflow rate; $C_{a}$ is its capacity; $x_{a, \max }$ is its maximum holding capacity.

\section{b. Actual Link Travel Time}

In practice, it is difficult to formulate the equation for actual link travel time due to the tremendous factors that may impact the actual link travel time greatly, such as different time period we focused on, road condition, traffic signal, congestion, incident, construction, weather, special events, and so on. As mentioned above, this property is called the stochastic characteristic of traffic condition (link travel time). However, in order to provide a reasonable approximation of actual link travel time, some researchers using certain distribution to represent the stochastic part 
of link travel time. Normal Distribution was applied in Liu and Ban's paper ${ }^{[9]}$ as shown in Equation (2) and (3):

$$
\begin{aligned}
& \tau_{a}(t)=c_{a}(t)+\xi_{a}(t) \\
& \xi_{a}(t) \sim N\left(\mu, \sigma^{2}\right)
\end{aligned}
$$

where $\tau_{a}$ is actual link travel time and $\xi_{a}$ is its stochastic term.

In this paper, equation (1), (2), (3) is used to estimate actual link travel time.

\section{c. Fuzzy Sets of Perceived Link Travel Time}

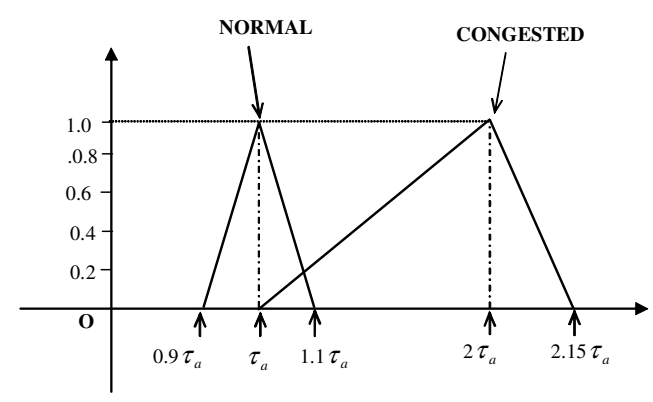

Figure 8. Membership function for fuzzy sets of perceived link travel time

\section{Fuzzy Sets for Perceived Link Travel Time}

Based on the actual link travel time, we can construct fuzzy sets for perceived link travel time. In this paper, we use linguistic descriptions to represent the fuzzy sets. Due to driver's experience of similar links, several linguistic descriptions of perceived link travel time can be formulated as below:

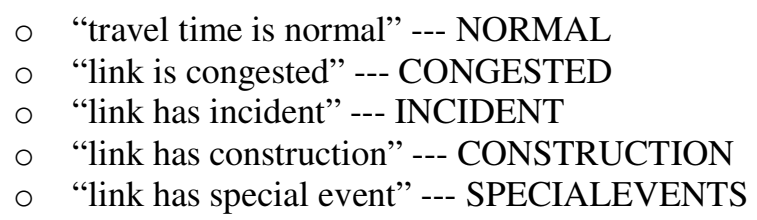

These fuzzy sets represent different level of traffic conditions and more sets may be added if necessary.

\section{Membership Function for Fuzzy Sets of Perceived Link Travel Time}

Generation of membership functions arouses a lot of interests in the literature. However, no standard method exists yet. The membership functions can be constructed based on a survey. To model driver's perceptions, in most applications of fuzzy set theory, TFN (Triangular Fuzzy Numbers) or TrFN (Trapezoidal fuzzy Numbers) are usually used. In this paper, triangular 
function is used to form the membership functions of the fuzzy sets in 2-3-1. Figure 13 shows the membership functions for NORMAL and CONGESTED fuzzy sets.

\section{Fuzzy Rules for Perceived Link Travel Time}

Only one simple rule will be used for these fuzzy sets of link travel time. "If the travel time of link A is less than that of link B, link A will be chosen." This rule implies that if link A is faster than link B, then link A, instead of link B, will be used by the driver.

\section{d. Fuzzy Sets of Perceived Path Travel Time}

In crisp case, path travel time equals to the summation of the travel time of those links that construct this path. However, for fuzzy case, we need to define how to formulate fuzzy sets of perceived path travel time based on fuzzy sets of perceived link travel time. Therefore, here we need to define fuzzy addition formulation.

In this paper, Dubois and Prade's method is used. Four steps are needed to perform fuzzy addition operation: Flattening, Decomposition, Operation * and Union. Here operation * is operation + . Generally, Dubois's method is a little complex to compute. But for fuzzy sets with triangular membership function, it is quite simple.

As shown in Figure 9, if we represent fuzzy set $\mathbf{S}$ that has triangular membership as $\mathbf{S}$ (L, M, R), its membership function can be expressed as:

$$
\mu_{A}(x)=\left\{\begin{array}{cc}
\frac{x-L}{M-L}, & x \in[L, M] \\
\frac{R-x}{R-M}, & x \in(M, R] \\
0, & x \notin[L, R]
\end{array}\right.
$$

And from Dubois's theory ${ }^{[12]}$, the addition of fuzzy set $S_{1}\left(L_{1}, M_{1}, R_{1}\right)$ and $S_{2}\left(L_{2}, M_{2}, R_{2}\right)$ will be:

$$
S_{1+2}=S_{1}+S_{2}=\left(L_{1}+L_{2}, M_{1}+M_{2}, R_{1}+R_{2}\right)
$$

From equation (4) and (5), we can easily get fuzzy path travel time and its membership function given the fuzzy travel time of those links that form this path. 

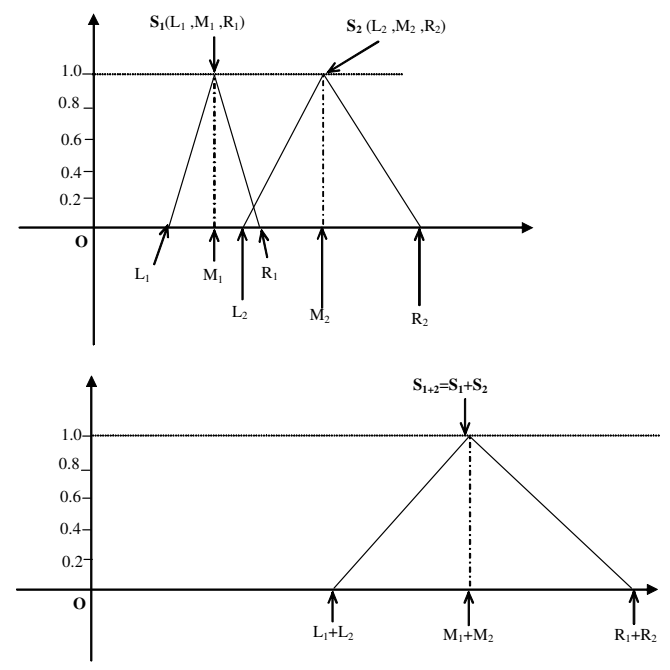

Figure 9. Fuzzy Addition Illustration

\section{e. Fuzzy Shortest Path (FSP) Algorithm}

Under crisp case circumstances, after we find the travel time for paths connecting origin and destination, we can find the shortest path and assign all traffic to this path. However, under fuzzy case, it is difficult to find only path that is the "shortest" in term of fuzzy travel time. Therefore, we need some method to find a group of fuzzy "shortest" paths and assign traffic to all of them based on their membership functions.

Several fuzzy shortest path algorithms have been proposed for solving the traffic assignment problem. In this paper, Blue's method is used.

According to Blue's classification, the road networks we are working on are actually a pure Type $\mathrm{V}$ fuzzy graph with crisp nodes and links as well as fuzzy weights (travel time). Denote $\mathrm{G}$ is a Type V fuzzy graph, we can find the fuzzy shortest paths using the following three steps.

\section{Step 1: Converting Fuzzy Graph to Crisp Graph}

We first construct two crisp graphs $\bar{G}$ and $\underline{G}$ that are identical to $\mathrm{G}$, except that the link travel time is crisp. Link travel time for graph $\bar{G}$ is:

$$
\bar{\tau}_{a}=\sup \left\{\operatorname{supp}\left(\tau_{\mathrm{a}}\right)\right\}
$$

where sup means least upper bound, and supp means support of fuzzy set.

Equation (7) is the link travel time for graph $\underline{G}$ :

$$
\underline{\tau}_{a}=\inf \left\{\operatorname{supp}\left(\tau_{\mathrm{a}}\right)\right\}
$$

where inf means greatest lower bound. 


\section{Step 2: Find Shortest Path in Graph $\bar{G}$}

Find the shortest path in graph $\bar{G}$. It is a traditional problem to find the shortest path in a crisp graph and various algorithms are available to solve it ${ }^{[14]}$. Denote $\mathrm{K}$ is the length of this shortest path.

\section{Step 3: Calculate Membership}

Denote $\underline{S}$ is the set of paths in $\underline{G}$, connecting origin to destination and with the length less than K.

Denote $S$ is a set of fuzzy paths in G. All paths in $S$ are identical to those paths in $\underline{S}$ respectively, except that the travel time is fuzzy. $S$ is the set of fuzzy shortest paths found and will be assigned traffic. Calculate the membership of $\mathrm{K}$ for each of the fuzzy path in $\mathrm{S}$.

\section{Fuzzy Traffic Assignment}

From 4, we can get the set of fuzzy shortest paths $\mathrm{S}$. We assume that $\mathrm{S}$ has $\mathrm{M}$ elements as displayed below:

$S=\left\{p_{1}, p_{2}, \ldots . p_{m}, \ldots p_{M}\right\}$

where $p_{m}$ is the fuzzy shortest path.

And the memberships of $\mathrm{K}$ in those fuzzy shortest paths are:

$$
f\left(p_{m}\right)=f_{m} \quad m=1,2, . . M
$$

Further, we assume the traffic flow from origin $\mathrm{O}$ to destination $\mathrm{D}$ is $T_{o d}$. In this paper, we assign the traffic flow to these paths in S proportionally to their membership using equation (10):

$$
T_{o d}^{m}=\frac{f_{m}}{\sum_{i=1}^{M} f_{i}}
$$

For dynamic traffic assignment (DTA), we have to consider the flow propagation. In this case, equation (11) is used for this purpose:

$\left.x_{a p}^{r s}(t)=\sum_{b \in \tilde{p}}\left\{x_{b p}^{r s}\left[t+\bar{\tau}_{a}(t)\right]-x_{b p}^{r s}(t)\right\}+\left\{E_{p}^{r s}\left[t+\bar{\tau}_{a}(t)\right]-E_{p}^{r s}(t)\right]\right\}$

where $p$ is the path a belongs to, $\tilde{p}$ is the subpath from a to destination D. 
International Journal on Soft Computing (IJSC) Vol.3, No.2, May 2012

\section{CONCLUSION}

Many problems encountered when studying complex transportation systems are highly nonlinear. As already noted, a fuzzy logic system is a nonlinear system that maps a crisp input vector into a crisp scalar output. When solving a large number of different traffic and transportation problems, this is what we actually do: map a crisp input vector into a crisp scalar output.

Fuzzy logic could be used successfully to model situations in which people make decisions in an environment that is so complex that it is very hard to develop a mathematical model. Such situations for example often occur in the field of traffic and transportation when studying the work of dispatchers or modeling choice problems. Present experience shows that there is room for the development of different approximate reasoning algorithms when solving complex problems of this type. Until a few years ago the 'trial and error' procedure was the customary method used to develop fuzzy logic systems, and so researchers designed fuzzy logic systems independently of numerical training data. Wang and Mendel (1992a,b) [46, 47]; Jang (1992) [13] and Horikawa et al. (1992) [11] proposed separately from each other to tune fuzzy logic system parameters using numerical training data.

This idea was accepted by a great many researcher and in the past two or three years a large number of papers have appeared in which the fuzzy rule base and/or shape of the membership function are determined using numerical training data. Designing fuzzy logic systems using numerical training data is certainly one of the very important tasks in the area of fuzzy logic systems for transportation engineering as well.

In the past several years, significant theoretical results have been achieved in the field of fuzzy logic systems. Wang and Mendel (1992c) [48] and Kosko (1992) [21]showed that fuzzy logic systems are universal approximators, which means that a fuzzy logic system 'can uniformly approximate any real continuous nonlinear function to an arbitrary degree of accuracy' (Mendel, 1995 [25]). This existence theorem shows us above all the possibilities offered by fuzzy logic, but does not indicate the manner in which to create fuzzy logic systems. In other words, the number of inputs, the number of fuzzy sets used to describe fuzzy variables, and the number of rules essentially influence the quality of the solution generated by a fuzzy logic system. It should be emphasized that feed-forward neural networks and fuzzy logic systems are techniques that can be used to solve the same class of problems. Since fuzzy logic system parameters can be initialized using expert knowledge, while weights in feed-forward neural networks are most often initialized randomly, fuzzy logic systems are tuned much more quickly than the tuning of feed-forward neural networks.

Fuzzy logic systems provide two other very important advantages. They can use existing linguistic knowledge very successfully, and they treat uncertainty in an appropriate manner.

The basic goal of this paper was to classify and analyze results in the application of fuzzy logic when modeling complex traffic and transportation processes. The results obtained show that fuzzy set theory and fuzzy logic present a promising mathematical approach to model complex traffic and transportation processes that are characterized by subjectivity, ambiguity, uncertainty and imprecision. As already noted, the benefits from the fuzzy logic will be more accurately assessed as the number of successful practical applications of the fuzzy logic in traffic control and transportation planning increases. 


\section{REFERENCES}

1. Akiyama, T., Shao, C.-F., (1993) "Fuzzy mathematical programming for traffic safety planning on an urban expressway" Transportation Planning and Technology, Vol. 17, pp. 179-190.

2. Akiyama, T., Yamanishi, H., (1993) "Travel time information service device based on fuzzy sets theory”, In: Ayyub, B.M. (Ed.), Proceedings of ISUMA '93, The Second International Symposium on Uncertainty Modeling and Analysis. IEEE Computer Press, College Park, Maryland, pp. 238-245.

3. Akiyama, T., Shao, C-F., Sasaki, T., (1994) "Traffic flow on urban networks with fuzzy information", Memorial Faculty of Engineering, Kyoto University, Vol. 56, pp. 1-22.

4. Akiyama, T., Tsuboi, H., (1996) "Description of route choice behaviour by multi-stage fuzzy reasoning", Paper presented at the Highways to the Next Century Conference, Hong Kong.

5. Chakroborthy, P., (1990) "Application of fuzzy set theory to the analysis of capacity and level of service of highways" MSc. thesis, University of Delaware, Newark, DE.

6. Chakroborthy, P., Kikuchi, S., (1990) "Application of fuzzy set theory to the analysis of capacity and level of service of highways" In: Ayyub, B.M. (Ed.), Proceedings of ISUMA '90, The First International Symposium on Uncertainty Modeling and Analysis. IEEE Computer Press, College Park, Maryland, pp. 146-150.

7. Chanas, S., Delgado, M., Verdegay, J.L., Vila, M.A., (1993) "Interval and fuzzy extensions of classical transportation problems", Transportation Planning and Technology Vol. 17, pp. 203-218.

8. Chen, L., May, A., Auslander, D., (1990) "Freeway ramp control using fuzzy set theory for inexact reasoning", Transportation Research, Vol. 24A, pp. 15-25.

9. Chang, Y.H., Shyu, T.H., (1993) "Traffic signal installation by the expert system using fuzzy set theory for inexact reasoning", Transportation Planning and Technology, Vol. 17, pp. 191-202.

10. Deb, S.K., (1993) "Fuzzy set approach in mass transit mode choice", In: Ayyub, B.M. (Ed.), Proceedings of ISUMA '93, The Second International Symposium on Uncertainty Modeling and Analysis. IEEE Computer Press, College Park, Maryland, pp. 262-268.

11. Horikawa, S., Furahashi, T., Uchikawa, Y., (1992) "On fuzzy modeling using fuzzy neural networks with back-propagation algorithm”, IEEE Transactions on Neural Networks, Vol. 3, pp. 801-806.

12. Hsiao, C.-H., Lin, C.-T., Cassidy, M., (1994) "Application of fuzzy logic and neural networks to automatically detect freeway traffic incidents", Journal of Transportation Engineering, Vol. 120, pp. 753-772.

13. Jang, J.-S.R., (1992) "Self-learning fuzzy controllers based on temporal back-propagation", IEEE Transactions on Neural Networks, Vol. 3, pp. 714-723.

14. Jassbi J., Makvandi P., Ataei M. and Sousa Pedro A. C., (2011) "Soft system modeling in transportation planning:Modeling trip flows based on the fuzzy inference system approach", African Journal of Business Management, Vol. 5(2), pp. 505-514.

15. Kalic Â ,M., Teodorovic $\hat{A}$, D., (1996) "Solving the trip distribution problem by fuzzy rules generated by learning from examples”, Proceedings of the XXIIIYugoslav SymposiumonOperationsResearch, Zlatibor, Yugoslavia, pp. 777-780 (in Serbian).

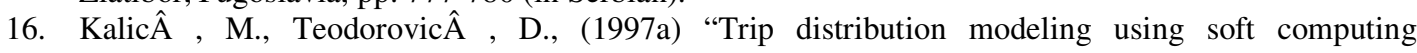
techniques", Paper presented at the EURO XV/INFORMS XXXIV (Book of abstracts, p. 74), Barcelona.

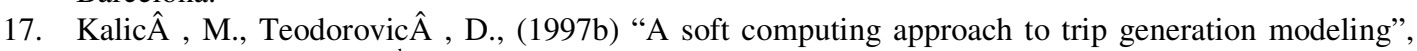
Paper presented at the $9^{\text {th }}$ Mini EURO Conference Fuzzy sets in traffic and transport systems, Budva, Yugoslavia.

18. Kikuchi, S., (1992) "Scheduling demand-responsive transportation vehicles using fuzzy-set theory", Journal of Transportation Engineering, Vol. 118, pp. 391-409.

19. Kikuchi, S., VukadinovicÂ , N., Easa, S., (1991) "Characteristics of the fuzzy LP transportation problem for civil engineering applications”, Civil Engineering Systems, Vol. 8, pp. 134-144.

20. Kikuchi, S., Perincherry, V., Chakroborthy, P., Takahashi, H., (1993) "Modeling of driver anxiety during signal change intervals", Transportation Research Record, Vol. 1399, pp. 27-35.

21. Kosko, B., (1992) "Fuzzy Systems as universal approximators", Proceedings of 1st IEEE International Conference on Fuzzy Systems, pp. 1153-1162.

22. Lotan, T., Koutsopoulos, H., (1993a) "Route choice in the presence of information using concepts from fuzzy control and approximate reasoning", Transportation Planning and Technology, Vol. 17, pp. 113-126. 
23. Lotan, T., Koutsopoulos, H., (1993b) "Models for route choice behaviour in the presence of information using concepts from fuzzy set theory and approximate reasoning" Transportation, Vol. 20, pp. 129-155.

24. Mamdani, E., Assilian, S., (1975) "An experiment in linguistic synthesis with a fuzzy logic controller" International Journal of Man-Machine Studies, Vol. 7, pp.1-13.

25. Mendel, J., (1995) "Fuzzy logic systems for engineering", A tutorial. Proceedings of the IEEE 83, pp. 345-377.

26. MilosavljevicÂ, N., TeodorovicÂ, D., PapicÂ, V., PavkovicÂ, G., (1996) "A fuzzy approach to the vehicle assignment problem", Transportation Planning and Technology Vol. 20, pp. 33-47.

27. Nakatsuyama, M., Nagahashi, N., Nishizuka, N., (1983) "Fuzzy logic phase controller for traffic functions in the one-way arterial road", Proceedings IFAC 9th Triennial World Congress. Pergamon Press, Oxford, pp. 2865-2870.

28. Pappis, C., Mamdani, E., (1977) “A fuzzy controller for a traffic junction”, IEEE Transactions on Systems, Man and Cybernetics SMC-7, pp. 707-717.

29. Perincherry, V., (1990) "Application of fuzzy set theory to linear programming" MSc. thesis, University of Delaware, Newark, DE.

30. Perincherry, V., Kikuchi, S., (1990) "A fuzzy approach to the transshipment problem”, In: Ayyub, B.M. (Ed.), Proceedings of ISUMA '90, The First International Symposium on Uncertainty Modeling and Analysis. IEEE Computer Press, College Park, Maryland, pp. 330-335.

31. Perkinson, D., (1994) "Using automated vehicle location data to monitor congestion,: Fuzzy set theory, ITE Journal, pp. 35-40.

32. Quadrado, J.C., Quadrado, A.F., (1996) "Fuzzy modeling of accessibility: Case study-Lisbon metropolitan area", Proceedings of the Fourth European Congress on Intelligent Techniques and Soft Computing,Aachen, Germany, pp. 1307-1311.

33. Sasaki, T., Akiyama, T., (1986) "Development of fuzzy traffic control system on urban expressway", Preprints 5th IFAC/IFIP/IFORS International Conference in Transportation Systems, pp. 333-338.

34. Sasaki, T., Akiyama, T., (1987) "Fuzzy on-ramp control model on urban expressway and its extension”, In: Gartner, N.H., Wilson, N.H.M. (Eds.), Transportation and traffic theory. Elsevier Science, New York, pp. 377-395.

35. Sasaki, T., Akiyama, T., (1988) “Traffic control process of expressway by fuzzy logic,. Fuzzy Sets and Systems, Vol. 26, pp.165-178.

36. Sugeno, M., Nishida, M., (1985) "Fuzzy control of model car”, Fuzzy Sets and Systems, Vol. 16, pp. 103-113.

37. TeodorovicÂA, D., (1994) "Invited review: fuzzy sets theory applications in traffic and transportation", European Journal of Operational Research, Vol. 74, pp. 379-390.

38. Teodorovic $\hat{A}$, D., BabicÂ , O., (1993) "Fuzzy inference approach to the flow management problem in air traffic control", Transportation Planning and Technology, Vol.17, pp. 165-178.

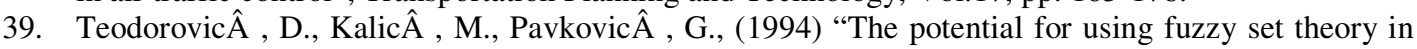
airline network design", Transportation Research 28B, pp.103-121.

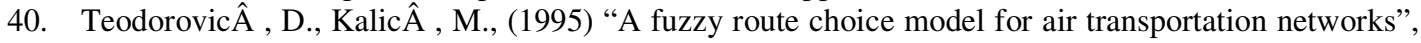
Transportation Planning and Technology Vol. 19, pp.109-119.

41. TeodorovicÂA, D., Kikuchi, S., (1990) “Transportation route choice model using fuzzy inference technique”, In: Ayyub, B.M. (Ed.), Proceedings of ISUMA '90, The First International Symposium on Uncertainty Modeling and Analysis. IEEE Computer Press, College Park, Maryland, pp. 140-145.

42. Teodorovic Â, D., Kikuchi, S., (1991) "Application of fuzzy sets theory to the saving based vehicle routing algorithm”, Civil Engineering Systems, Vol. 8, pp. 87-93.

43. TeodorovicÂA, D., PavkovicÂA, G., (1996) The fuzzy set theory approach to the vehicle routing problem when demand at nodes is uncertain. Fuzzy Sets and Systems Vol. 82, pp. 307-317.

44. Tzeng, G.-H., TeodorovicÂA, D., Hwang, M-J., (1996) "Fuzzy bicriteria multi-index transportation problems for coal allocation planning of Taipower", European Journal of Operational Research, Vol. 95, pp. 62-72.

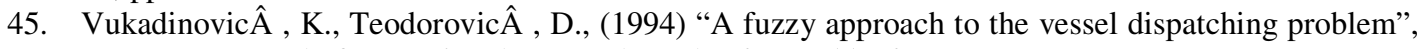
European Journal of Operational Research, Vol. 76, pp.155-164.

46. Wang, L-X., Mendel, J., (1992a) "Generating fuzzy rules by learning from examples", IEEE Transactions on systems, Man and Cybernetics, Vol. 22, pp. 1414-1427. 
47. Wang, L-X., Mendel, J., (1992b) "Back-propagation of fuzzy systems as nonlinear dynamic system identifiers", Proceedings IEEE International Conference on Fuzzy Systems, San Diego, CA, pp. $807-$ 813.

48. Wang, L.-X., Mendel, J., (1992c) "Fuzzy basis functions, universal approximation, and orthogonal least squares learning", IEEE Transactions on Neural Networks, Vol.3, pp. 807-813.

49. Xu, W., Chan, Y., (1993a) "Estimating an origin-destination matrix with fuzzy weights", Part 1: Methodology. Transportation Planning and Technology, Vol. 17, pp. 127-144.

50. Xu, W., Chan, Y., (1993b) "Estimating an origin-destination matrix with fuzzy weights", Part 2: Case studies. Transportation Planning and Technology, Vol. 17, pp. 145-164.

51. Zadeh, L.,(1973) "Outline of a new approach to the analysis of complex systems and decision processes”, IEEE Transactions on Systems, Man and Cybernetics SMC-3, pp. 28-44.

\section{Authors:}

\begin{abstract}
Amrita Sarkar
Amrita Sarkar is a graduate Engineer in Information Technology with a post graduation in Remote Sensing. She is presently a PhD Research Fellow at the Department of Information Technology, Mesra, India. She has got few research publications in her area of specialization. Her areas of interests include Soft Computing, Artificial Intelligence, Data Mining, DBMS and Image Processing.
\end{abstract}

\section{Dr. G. Sahoo}

Dr. G. Sahoo received his MSc in Mathematics from Utkal University in the year 1980 and $\mathrm{PhD}$ in the area of Computational Mathematics from Indian Institute of Technology, Kharagpur in the year 1987. He has been associated with Birla Institute of Technology, Mesra, Ranchi, India since 1988, and currently, he is working as a Professor and Head in the Department of Information Technology. His research interest includes theoretical computer science, parallel and distributed computing, evolutionary computing, information security, image processing and pattern recognition.

\section{Dr. U. C. Sahoo}

Dr. U. C. Sahoo is working as an Assistant Professor in the Department of Civil Engineering, Indian Institute of Technology, Bhubaneswar and is an expert in the field of Transportation Engineering. He has more than eight years of teaching and research experience. Presently he is engaged in research in the area of transportation planning, road safety and pavement engineering and published many papers in these areas.
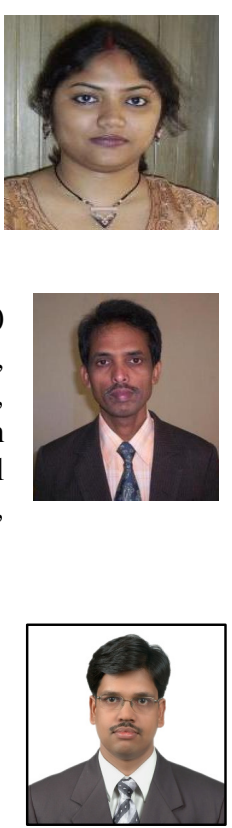